\title{
DECODING THE RHYTHMS OF THE NATURAL WORLD
}

PATTERNS ARE EVERYWHERE IN NATURE, FROM SEASONS TO COLOUR SCHEMES TO HEARTBEATS. THE MORE YOU LOOK, THE MORE YOU FIND, AND THE MORE COMPLEX THEY BECOME. DR BARD ERMENTROUT OF THE UNIVERSITY OF PITTSBURGH USES ADVANCED MATHEMATICS TO DECIPHER THESE PATTERNS

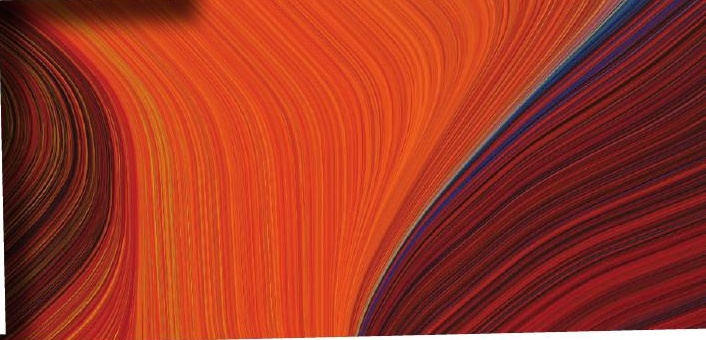

\section{TALK LIKE A MATHEMATICAL BIOLOGIST}

\section{EPIDEMIOLOGY}

The study of how diseases spread and can be controlled.

\section{LINEAR MODEL}

A model is linear if changes in the inputs result in proportional outputs of the model.

\section{NON-LINEAR MODEL}

A mathematical model that does not follow a straight line on a graph. A model is non-linear if it is not linear!

\section{PHENOMENA}

Something observed, like a situation or event.

\section{SPATIAL}

Related to space.

\section{TEMPORAL}

Related to time.

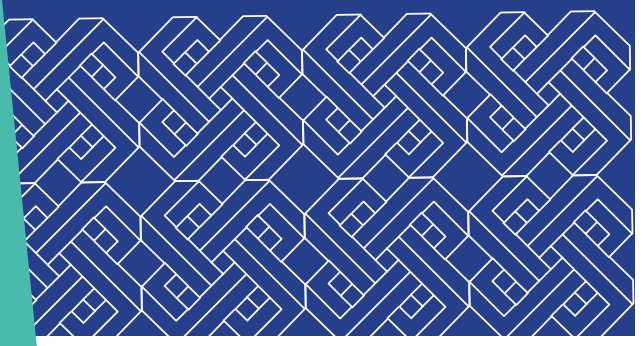

The natural world is a complex place and being able to understand it requires complex tools. Dr Bard Ermentrout, Distinguished University Professor at the University of Pittsburgh in the USA, has plenty of expertise in applying these tools and learning exactly what the natural world has to tell us. Two of his recent projects involve understanding 'flicker hallucinations' and the mechanisms behind seashell colouration patterns.

\section{FLICKERS OF UNDERSTANDING}

Have you ever noticed how geometric shapes can appear in your field of vision when you're looking at flickering light? These 'flicker hallucinations' form an illusory pattern in your visual field, and Bard has undertaken research to understand exactly how and why this takes place. "Maths provides a framework for understanding many natural phenomena, and very different instances can lead to similar mathematical patterns," he says.

In a very different scenario, if you add oil to a warming frying pan and then evenly add pepper or paprika, the spice will separate into distinct geometric structures. Mathematical biologists call this 'spontaneous symmetry breaking'. It is the same general process that happens when the light uniformly hitting our eyes appears to break into distinct geometric shapes. "In terms of physics, there are big differences, but it's the same underlying mathematics," says Bard.

These processes occur when something 'loses stability'; it changes from a normal state (like the everyday function of our eyes) into something else. You can achieve this effect with all kinds of things. For instance, if moved, a pendulum will swing back and forth until it comes to rest. However, if you move the base of the pendulum a certain way at the same time, it will give the appearance of going haywire. It is the same principle as swinging your legs back and forth to go higher on a swing. Using mathematics to understand these unstable states when they occur in biology can reveal interesting secrets.

\section{BREAKING IT DOWN}

Studying flicker hallucinations can be challenging because the shapes are difficult to control and report. Bard's team managed to simplify the effect by reducing the flickering light to a thin ring. They were then able to quantify the effects they saw, ending up with data that could be mathematically processed.

Psychologists were also brought in to understand certain phenomena. "We noticed that the patterns on the ring of flickering light appeared to rotate, first one way and then the other," says Bard. Understanding how the brain processes visual information is critical to uncovering why these odd effects are seen.

So, what's the purpose of this research? One theory is that these shapes provide a window into early visual systems, in terms of in the first processing units in the cortex (the main part of our brains). These systems would interpret light or darkness as a simple 'on or off', which is perhaps why certain flickering lights appear to us as block geometric shapes. The patterns that we see are directly related to the way that the neurons are connected in this part of the 



\section{ABOUT}

\section{MATHEMATICAL BIOLOGY}

Mathematical biology involves the construction of mathematical models to answer biological questions. To take a simple example, say that we know a crocodile increases in length by $\mathrm{x} \mathrm{cm}$ every year (they continue growing throughout their lives, after all). How do we predict how long it will be in several years' time? This involves constructing a formula, in this case $I=x y$, where $l$ is length and $y$ is the number of years since it was born.

This formula is a very simple model of linear dynamics. If you drew it on a graph of length against time, it would be a straight line. Most biological processes are far more complex than that, especially when it comes to patterns in nature. Bard specialises in non-linear dynamics. "Non-linear dynamics are much more complicated because there are many possible states and paths, depending on where you start," he says. On the crocodile example, he expands, "As the crocodile grows in length, his girth also grows and, in fact, grows in a way that is proportional to his length; if his length doubles, his girth doubles. On the other hand, his weight will increase non-linearly, basically like his length cubed (since volume is proportional to length^3)."

\section{DETECTING A PATTERN}

Nature is full of patterns. Bard is interested in patterns that vary over space (such as spots or stripes) or time (such as rhythm). When we think of patterns, we most naturally think of spatial patterns, of which there are plenty of examples in the animal kingdom. Patterns that mimic an animal's surroundings, such as a tiger's stripes, help with camouflage, while patterns that stand out often act as a warning of danger. Recognition of these patterns can be deeply encoded within DNA. For instance, some birds will instinctively be afraid of a colourful snake, even if they have never seen a snake before in their life.

Temporal patterns are those that play out over time. For instance, think of a horse. When it is walking slowly, it moves one leg at a time; when trotting it moves diagonally opposite legs at once; and at a gallop, only one hoof touches the ground at a time. These are temporal patterns. "Gaits must be able to deal with uneven surfaces and obstacles, and using set patterns helps to overcome these," says Bard. We have our very own temporal patterns for walking, running, cycling, rowing and any other repetitive activity that our bodies undertake.

\section{MATHS ON (AND IN) THE BRAIN}

Bard takes a special interest in applying his expertise to neuroscience. "Many neuroscientists are interested in mechanisms, perhaps more so than most other biologists," he says. "We have new experimental methods that allow us to tackle problems that would have been unimaginable before. And, of course, the brain and nervous system are just very cool."

The human nervous system can be affected by patterns in odd ways. "When looking at a flashing light (a temporal pattern), some people can experience migraines or even seizures," says Bard. "In most, we will see geometric patterns called flicker phosphenes." Optical illusions provide another example: for instance, we might see flashing dots in highcontrast patterns, when no dots are actually there. Building models of why these effects happen is one of Bard's areas of interest.

\section{THE MAKINGS OF A MATHEMATICIAN}

"My aim is to provide explanations or

mechanisms for things we observe in the natural world," says Bard. Being able to do this requires a comprehensive knowledge of modelling, and also the tools to put these into practice. "You need to choose the right tool, and the tools available are always expanding," he says. Models involve a simplification of a complex, messy system, and making sure that you simplify in the right direction is crucial. As Einstein said, "A model should be as simple as it can be but no simpler." Collaboration is also vitally important. Biologists tend not to have an in-depth knowledge of mathematics, and vice versa. Combining unique sets of skills is a cornerstone of good science.

\section{HOW TO BECOME A MATHEMATICAL BIOLOGIST}

- There are some undergraduate degrees available in mathematical biology, but more common degrees such as mathematics and, sometimes, biology can also lead to a career in this area.

- According to The Complete University Guide, the top UK universities for mathematics are Oxford, Cambridge, St Andrews, Durham and Imperial College London.

- According to Top Universities, the top US universities for mathematics are MIT, Harvard, Stanford, California Berkeley and Princeton. Bard advises that the best universities for mathematical biology are less well-known and include Utah, Michigan, Columbia and New York University.

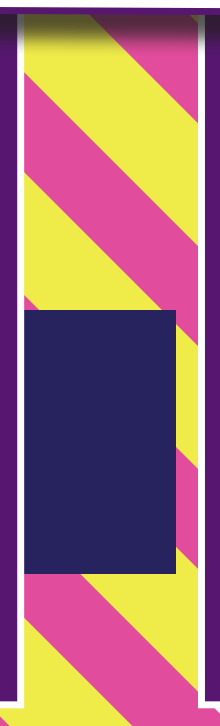

\section{PATHWAY FROM SCHOOL TO MATHEMATICAL BIOLOGIST}

At school, mathematics, further mathematics, biology and other sciences are advantageous. At university, Bard recommends taking classes in calculus and linear algebra, and, if possible, basic science, probability and statistics, and differential equations.

Programming and coding ability will also prove useful. Bard advises learning LaTeX, the digital tool used for writing mathematical formulae and models, as well as some sort of higher-level programming language like MatLab or Python. 
